\title{
Intravenous Carbon Dioxide as an Echocardiographic Contrast Agent
}

\author{
Richard S. Meltzer, MD, Patrick W. Serruys, MD, \\ Paul G. Hugenholtz, MD, and Jos Roelandt, MD
}

\begin{abstract}
Intravenous carbon dioxide $\left(\mathrm{CO}_{2}\right)$ was employed to cause echocardiographic contrast in 40 patients. One to $3 \mathrm{cc}$ of medically pure $\mathrm{CO}_{2}$ were agitated with 5 to $8 \mathrm{cc}$ of $5 \%$ dextrose in water and rapidly injected into an upper extremity vein. Contrast was obtained in all patients. In 33 patients contrast density from $5 \%$ dextrose was compared with that from $5 \%$ dextrose- $\mathrm{CO}_{2}$ injections. Six of these patients had no contrast on the initial 5\% dextrose injection and definite contrast with the subsequent injection containing $\mathrm{CO}_{2}$. Of the 33,12 patients had initial contrast with $5 \%$ dextrose injections and greater contrast density when $\mathrm{CO}_{2}$ was added; 15 showed no definite difference; and none had less contrast with intravenous $\mathrm{CO}_{2}-5 \%$ dextrose than with $5 \%$ dextrose alone. Intravenous $\mathrm{CO}_{2}-5 \%$ dextrose is a useful method of increasing contrast in those patients who fail to demonstrate echocardiographic contrast when routine techniques are employed. It is also safe, provided precautions emphasized in this paper are observed. Indexing Words: Echocardiography · Ultrasound contrast · Carbon dioxide
\end{abstract}

Since Gramiak et al $^{1}$ first described ultrasonic contrast over a decade ago, contrast echocardiography has achieved widespread clinical application. ${ }^{2-6}$ However, both the fluid employed and the injection techniques used vary widely among clinical echocardiography laboratories. As a result, a reliable and reproducible contrast effect is not always achieved. A 1975 textbook of echocardiography states: "Development of an ideal contrast agent is particularly important for the advancement of cardiac ultrasound . . . [it should] be administered noninvasively, be harmless and well tolerated, and produce a contrast effect that is consistent and reproducible. Development of such an ideal agent represents one of the important goals of echocardiographic research." ${ }^{6}$

Many echocardiography laboratories use in-

From the Thoraxcenter, Department of Clinical Echocardiography, Erasmus University and University Hospital Dijkzigt, Rotterdam, the Netherlands. Supported in part by grants from the Dutch Heart Association and Interuniversity Cardiology Institute. Manuscript received May 7, 1980; revised manuscript accepted August 7, 1980. For reprints contact Richard S. Meltzer, MD, Thoraxcenter, AZR-Dijkzigt, Dr Molewaterplein 40, 3015 GD Rotterdam, the Netherlands. docyanine green solutions for their ultrasonic contrast studies. However, in our experience indocyanine green is expensive, must be freshly mixed, frequently causes stains, and cannot be used for many repeated studies.

Early echocardiographers thought that the microbubbles causing contrast came from catheter tip cavitation. ${ }^{7}$ More recent work suggests that these microbubbles originate from gas present in the fluid or injection apparatus prior to injection. ${ }^{8-9}$ Thus we felt that a safe method of increasing the gas-phase content of blood would probably increase its contrast content. Since intravenous carbon dioxide in small quantities is known to be safe, we decided to examine whether it could decrease the proportion of unsatisfactory echocardiographic contrast studies.

\section{METHODS}

The study population consisted of 40 patients undergoing contrast echocardiograms for routine clinical indications $(\mathrm{N}=15$ patients) or as part of a research protocol attempting to assess tricuspid regurgitation $(\mathrm{N}=25)$. The study included all persons having contrast echocardiograms during a 
two-month period, with the exception of five persons suspected of having a right-to-left shunt.

Contrast injections were performed through 18- or 19-gauge catheters or butterfly needles introduced into a superficial upper extremity vein. All injections were performed forcefully by hand using a 10-cc plastic syringe attached to a threeway stopcock. The first one or two injections were 5 to 8 cc of $5 \%$ dextrose in water, and subsequent injections were 4 to $7 \mathrm{cc}$ of $5 \%$ dextrose in water agitated with 1 to $3 \mathrm{cc}$ of $\mathrm{CO}_{2}$ immediately before injection. Only $100 \%$ (medically pure) $\mathrm{CO}_{2}$ was used and a strict sterile technique employed. Three to ten (mean six) contrast injections were performed per study, generally employing several different transducer positions and echocardiographic views, and usually using both M-mode and two-dimensional equipment. An Organon Teknika EchocardioVisor $01^{*}$ was used for M-mode studies, and an Organon Teknika EchocardioVisor 03 Fociscan linear array unit or a Toshiba SSH-10A phased array sector scanner $\dagger$ was used for the two-dimensional studies. On all studies receiver gain was set just under the threshold of intracavitary noise that may mask or mimic contrast.

Results were judged by subjectively comparing contrast density during the first injection with $\mathrm{CO}_{2}-5 \%$ dextrose to the density seen during the previous injection of $5 \%$ dextrose alone but with the same echocardiographic instrument and view. The two-dimensional echocardiographic view employed was most frequently the subcostal short axis view imaging the right atrium and inferior vena cava, ${ }^{10}$ since these studies were combined with a research protocol to assess the utility of contrast echocardiography in this view for diagnosing tricuspid regurgitation. The apical fourchamber and parasternal long axis views were each used on several patients to compare contrast density. Density was rated during the echo study by the consensus of two of the authors (RSM and JM) who were present for all studies. Disagreements were resolved by later review of the studies.

\section{RESULTS}

Six of the 40 patients had no contrast from the initial 5\% dextrose injection. All patients had ultrasonic contrast imaged in the right side of the

*Organon Teknika Corp, Box 19080, Oklahoma City, OK 73144 .

†Toshiba Medical Systems, 1154 Dominguez St, Carson, CA 90745. heart as a result of the first peripheral injections with $\mathrm{CO}_{2}-5 \%$ dextrose which followed the initial $5 \%$ dextrose injection(s). Only two injections with $\mathrm{CO}_{2}$-dextrose failed to yield contrast: These were the fifth and sixth injections with $\mathrm{CO}_{2}-5 \%$ dextrose in a subject whose intravenous line infiltrated, and subcutaneous crepitus could be palpated in the area of infiltration.

An average of six injections with $\mathrm{CO}_{2}-5 \%$ dextrose and $10 \mathrm{cc}$ total $\mathrm{CO}_{2}$ were administered per person. No one experienced any discomfort or any symptoms related to the contrast injections. There were no signs of new cardiorespiratory distress in any patient during the study.

Of the 40 patients, 33 had contrast studies where the initial injection of $\mathrm{CO}_{2}-5 \%$ dextrose employed the same view and ultrasonic apparatus as the $5 \%$ dextrose injection immediately preceding it. Of these, 15 had no definite change in contrast intensity. Twelve of the 33 had contrast on the initial 5\% dextrose injection but definite increase in contrast intensity with $\mathrm{CO}_{2}$-dextrose (Fig 1,2). The six without echocardiographic contrast on the initial 5\% dextrose injection all had contrast on the subsequent $\mathrm{CO}_{2}-5 \%$ dextrose study. We saw no instances in which the ultrasound intensity decreased from the $5 \%$ dextrose to the $\mathrm{CO}_{2}-5 \%$ dextrose.

\section{DISCUSSION}

During serial ultrasonic contrast injections, the contrast effect frequently seems to be influenced by prior injections. We believe this is due to the margination of microbubbles and perhaps macrobubbles of gas in the syringe and catheter along the course of an upper extremity vein after a contrast injection, and the "flushing" effect of a subsequent injection. Ultrasonic contrast can be induced by "milking" an upper extremity vein or elevating the arm long after a contrast injection. It is possible but unlikely that the contrast increase noted during the $\mathrm{CO}_{2}-5 \%$ dextrose injections relates to contrast introduced during the earlier 5\% dextrose injections. We did not test this hypothesis for the following two reasons. First, we feel it is safer to begin contrast studies with $5 \%$ dextrose alone, to exclude major right-to-left shunts before $\mathrm{CO}_{2}$ is used. Secondly, it would be difficult to do the study "blinded," since the physician can both see 1 to $3 \mathrm{cc}$ of $\mathrm{CO}_{2}$ in the syringe and hear it gurgle as it enters the vein during injection. Since our study was not blinded, we cannot entirely exclude bias as an explanation for our findings. However, a single observer (RSM) performed all the injections of both 5\% dextrose and 


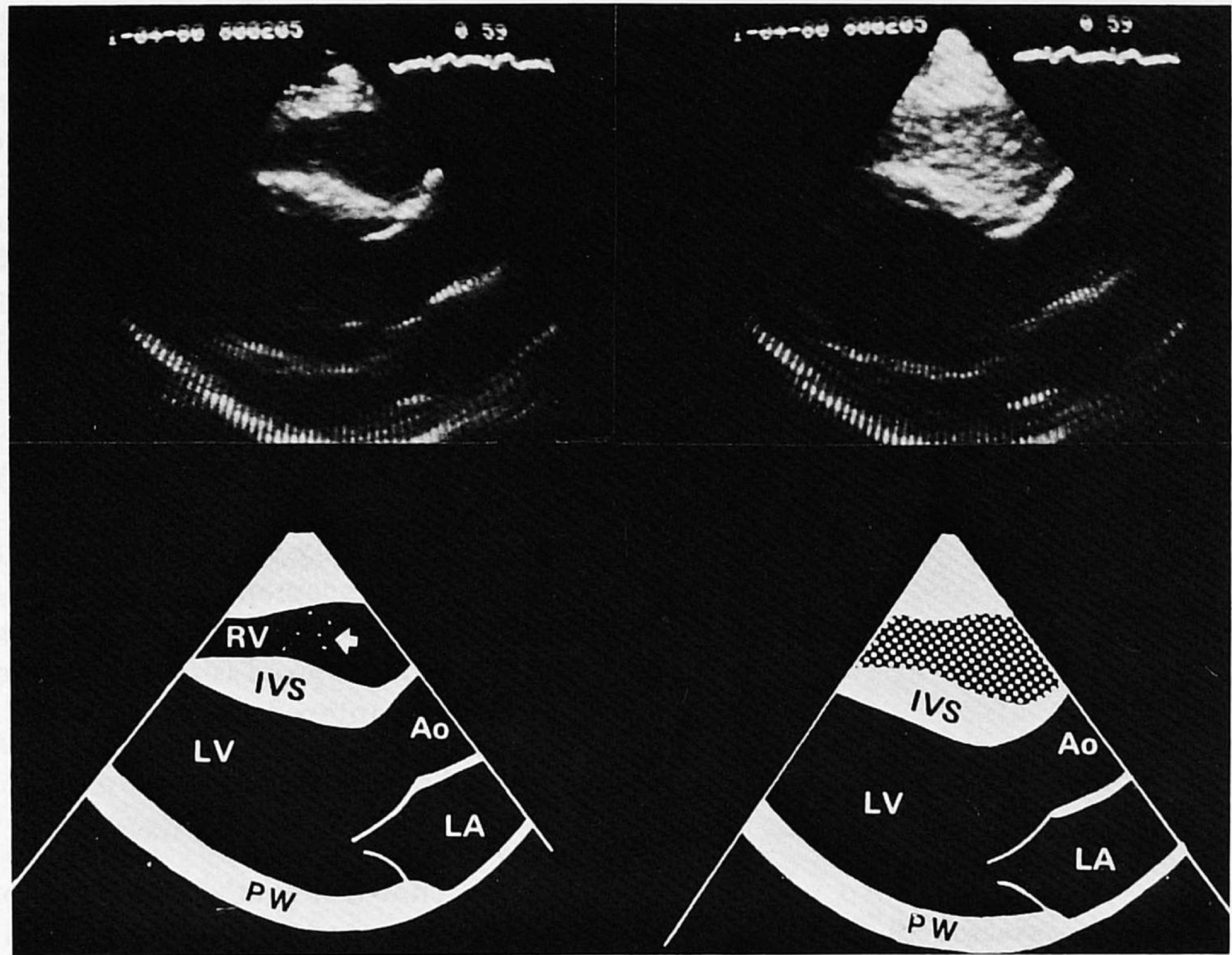

FIGURE 1. Upper panels: Polaroid photographs of stop-frame images from a two-dimensional echocardiographic study in a subject who had increased contrast density from the $5 \%$ dextrose injection (left) to the $\mathrm{CO}_{2}-5 \%$ dextrose injection (right). Parasternal long axis view. Lower panels: Diagrams of the photographs in the upper panels. Contrast in the right ventricle is indicated by arrow on the left and shaded area in the right-hand panel. RV: right ventricle. IVS: interventricular septum. LV: left ventricle. LA: left atrium. Ao: aorta. PW: posterior wall.

$\mathrm{CO}_{2}-5 \%$ dextrose and attempted to keep injection technique constant. We find it unlikely that the striking difference in contrast effect noted in most of the patients was due to bias alone.

Fifteen of our patients had no qualitative change in echo contrast from the initial 5\% dextrose injection to the subsequent $\mathrm{CO}_{2}-5 \%$ dextrose injections. This is probably because sufficient microbubbles were delivered to the right heart with the initial injection to completely fill the right heart with contrast. Further microbubbles in this situation may cause an "overload" effect and actually decrease the intensity of displayed contrast (and all anatomic structures) distal to the strongly reflecting microbubble layer. ${ }^{7}$

Echocardiographic contrast cannot at present be quantified, though some experimental approaches have been suggested that may lead to this goal in the future. ${ }^{8,11,12}$ The grading system in this study therefore had to be qualitative. This corresponds with the clinical state of the art in contrast echocardiology. Occasionally there is a need for qualitatively more intense contrast in a patient, but as yet quantification is not in routine clinical use.
The safety of intravenous $\mathrm{CO}_{2}$ had been established in the radiologic literature for diagnosing pericardial effusions, largely before the echocardiographic era. ${ }^{13-18}$ Considerably larger volumes of $\mathrm{CO}_{2}$ were injected (50 to $100 \mathrm{cc}$ ) than those necessary to create echocardiographic contrast. Emphasis on left lateral decubitus position and exclusion of patients with intracardiac shunts is found in several of these articles. Fortunately, most echocardiographic studies are performed in various degrees of left lateral decubitus position. Recently a large series of patients received $200 \mathrm{cc}$ of intravenous $\mathrm{CO}_{2}$ without detrimental effects. ${ }^{17}$ Blood chemistry does not change significantly with 1 -cc $\mathrm{CO}_{2} / \mathrm{kg}$ body weight, and experimental animals even tolerate $7.5 \mathrm{cc} / \mathrm{kg}$ of $\mathrm{CO}_{2}$ rapidly injected into the left ventricle or carotid artery with minimal cardiorespiratory effects. ${ }^{13}$ The same dose of air intravenously is fatal in most animals.

We feel that the smaller doses of $\mathrm{CO}_{2}$ employed to attain echocardiographic contrast in our patients are safe. Since data on intravenous $\mathrm{CO}_{2}$ in patients with right-to-left shunts do not exist, we would be cautious in its use in patients known to have shunts. Reale et al have recently reported on injections of 0.5 to $1 \mathrm{cc}$ of $\mathrm{CO}_{2}$ in the pulmonary 




FIGURE 2. M-mode echocardiograms recorded from a patient who was judged to have definite increase in right ventricular contrast density from the $5 \%$ dextrose injection (upper panel) to the following injection of $2 \mathrm{cc}$ of $\mathrm{CO}_{2}$ in $5 \mathrm{cc}$ of dextrose (lower panel). Injection technique and instrument settings were held constant. C: contrast. LV: left ventricle. IVS: interventricular septum. RV: right ventricle.

wedge position in humans. These injections yielded left heart echo contrast with no adverse effects observed. ${ }^{19}$

As pointed out above, a reliable method to achieve echo contrast would be desirable. In clinical practice, echocardiographic contrast techniques vary widely: Some laboratories use indocyanine green, others insist on small needles and specific stopcock arrangements, and some even tolerate the injection of small amounts of air. Part of this variability is due to a lack of theoretic understanding of the source of ultrasound contrast. We are convinced that the source is microbubbles present in the injecting fluid and apparatus rather than needle tip cavitation. ${ }^{8}$ Although elaborate right-angle stopcocks may cause turbulence and break up larger bubbles into microbubbles, small needles probably hinder the rapid flush effect necessary to deliver contrast to the central circulation and thus cause loss of microbubble content due to margination. Indocyanine green increases contrast content because it is a surfactant and stabilizes microbubbles. ${ }^{8}$ However, as mentioned, it is relatively expensive, must be used the same day it is prepared, contains a small amount of iodine which may cause allergic reactions or interfere with thyroid function tests, and frequently causes stains. Intravenous ether, carbonated water, and peroxide are other agents that cause echo contrast in small quantities, ${ }^{20}$ but their safety and practicality have not been evaluated in large groups of patients. There remains in clinical echocardiographic practice a small portion of patients in whom adequate contrast is difficult to obtain. Frequently these are patients with only distal veins available. Three of the six in our study whose initial injections failed to cause contrast had butterfly needles inserted in small hand veins. These may be the patients in whom $\mathrm{CO}_{2}$ contrast enhancement is most advantageous. Of course it is possible that in some patients even $\mathrm{CO}_{2}$ will fail to yield contrast, although we have not yet encountered such a patient.

\section{CONCLUSION}

We feel that a few cc's of intravenous medically pure $\mathrm{CO}_{2}$ is safe in persons with no right-toleft shunt. This procedure frequently increases echocardiographic contrast density and may be useful clinically in some patients. With further experience, $\mathrm{CO}_{2}$ might also provide a basis for quantitative contrast echocardiology, which is technically feasible and may yield important information in the future..$^{7,10,11}$

\section{ACKNOWLEDGMENTS}

The authors wish to thank Ellen Verheul and Anneke Wagenaar for their assistance in manuscript preparation and Jackie McGhie, Wim Vletter, and Willem Gorissen for their technical assistance.

\section{REFERENCES}

1. Gramiak R, Shah PM: Echocardiography of the aortic root. Invest Radiol 3:356, 1968.

2. Gramiak R, Shah PM, Kramer DH: Ultrasound cardiography: Contrast studies in anatomy and function. Radiology 92:939, 1969.

3. Kerber RE, Kioschos JM, Lauer RM: Use of an ultrasonic contrast method in the diagnosis of valvular regurgitation and intracardiac shunts. $A m J$ Cardiol 34:722, 1974.

4. Seward JB, Tajik AJ, Spangler JG, et al: Echocardiographic contrast studies: Initial experience. Mayo Clin Proc 50:163, 1975.

5. Hagemeijer F, Serruys PW, van Dorp WG: Contrast echocardiology, in Bom N (ed): Echocardiology. The Hague, Martinus Nijhoff, 1977, p 147.

6. Gramiak R, Nanda NC: Structure identification in 
echocardiography, in Gramiak R, Waag RC (eds): Cardiac Ultrasound. St. Louis, C.V. Mosby Co, 1975, p 29.

7. Kremkau FW, Gramiak R, Carstensen EL, et al: Ultrasonic detection of cavitation at catheter tips. Am J Roentgenol 110:177, 1970.

8. Meltzer RS, Tickner EG, Sahines TP, et al: The source of ultrasonic contrast. J Clin Ultrasound 8:121, 1980.

9. Barrera JG, Folkerson PK, Rittgers SE, et al: The nature of contrast echocardiographic "targets." Circulation (abstract) 57-58(suppl):II-233, 1978.

10. Meltzer RS, Roelandt J: Sector scanning views in echocardiology. Eur Heart $J$ (in press).

11. Bommer WJ, Mason DT, DeMaria AN: Studies in contrast echocardiography: Development of new agents with superior reproducibility and transmission through the lungs. Circulation (abstract) 59 60(suppl):II-17, 1979.

12. Bommer W, Lantz B, Miller L, et al: Advances in quantitative contrast echocardiography: Recording and calibration of linear time-concentration curves by videodensitometry. Circulation (abstract) 59-60 (suppl):II-18, 1979

13. Oppenheimer MJ, Durant TM, Stauffer HM, et al: In vivo visualization of intracardiac structures with gaseous carbon dioxide: Cardiovascular- respiratory effects and associated changes in blood chemistry. Am J Physiol 186:325, 1956.

14. Scatliff JH, Kummer AJ, Janzen AH: The diagnosis of pericardial effusion with intracardiac carbon dioxide. Radiology 73:871, 1959.

15. Stauffer HM, Durant TM, Oppenheimer MJ: Gas embolism: Roentgenologic considerations, including the experimental use of carbon dioxide as an intracardiac contrast material. Radiology 68:686, 1956.

16. Phillips JH, Burch GE, Hellinger R: The use of intracardiac carbon dioxide in the diagnosis of pericardial disease. Am Heart $J$ 61:748, 1961.

17. Turner AF, Meyers HL, Jacobson G, et al: Carbon dioxide cineangiocardiography in the diagnosis of pericardial disease. Am J Roent Rad Ther 97:342, 1966.

18. Bendib M, Toumi M, Boudjellab A: Carboxyangiographie et carboxyangiographie élargie en cardiologie. Ann Radiol 20:673, 1977.

19. Reale A, Pizzuto F, Gioffrè PA, et al: Contrast echocardiography: Transmission of echoes to the left heart across the pulmonary vascular bed. Eur Heart $J$ 1:101, 1980.

20. Ziskin MC, Bonakdarpour A, Weinstein DP, et al: Contrast agents for diagnostic ultrasound. Invest Radiol 7:500, 1972. 